\title{
THE IMPACT OF THE BOHR TOPOLOGY ON SELECTIVE PSEUDOCOMPACTNESS
}

\author{
DMITRI SHAKHMATOV AND VÍCTOR HUGO YAÑEZ
}

\begin{abstract}
Recall that a space $X$ is selectively pseudocompact if for every sequence $\left\{U_{n}: n \in \mathbb{N}\right\}$ of non-empty subsets of $X$ one can choose a point $x_{n} \in U_{n}$ for all $n \in \mathbb{N}$ such that the resulting sequence $\left\{x_{n}: n \in \mathbb{N}\right\}$ has an accumulation point in $X$. This notion was introduced under the name strong pseudocompactness by García-Ferreira and Ortiz-Castillo; the present name is due to Dorantes-Aldama and the first author. In 2015, García-Ferreira and Tomita constructed a pseudocompact Boolean group that is not selectively pseudocompact. We prove that if the subgroup topology on every countable subgroup $H$ of an infinite Boolean topological group $G$ is finer than its maximal precompact topology (the so-called Bohr topology of $H$ ), then $G$ is not selectively pseudocompact, and from this result we deduce that many known examples in the literature of pseudocompact Boolean groups automatically fail to be selectively pseudocompact. We also show that, under the Singular Cardinal Hypothesis, every infinite pseudocompact Boolean group admits a pseudocompact reflexive group topology which is not selectively pseudocompact.
\end{abstract}

As usual, $\mathbb{N}$ denotes the set of natural numbers and $\mathfrak{c}$ denotes the cardinality of the continuum. A group $G$ is Boolean if $x^{2}=e$ for every $x \in G$, where $e$ is the identity element of $G$. It is known (and easy to see) that all Boolean groups are abelian.

For a subset $X$ of a group $G$, the symbol $\langle X\rangle$ denotes the subgroup of $G$ generated by $X$; that is, the smallest subgroup of $G$ containing $X$.

A topological group $G$ is precompact, or totally bounded, provided that for every open neighbourhood $U$ of the identity of $G$ one can find a finite subset $F$ of $G$ such that $G=F U$. It is well known that a topological group $G$ is precompact if and only if it is a subgroup of some compact group. A classical result of Comfort and Ross [3] says that pseudocompact groups are precompact.

All topological groups in this paper are assumed to be Hausdorff.

\section{INTRODUCTION}

Recall that a point $x$ of a topological space $X$ is an accumulation point of a sequence $\left\{x_{n}: n \in \mathbb{N}\right\}$ of points of $X$ provided that the set $\left\{n \in \mathbb{N}: x_{n} \in V\right\}$ is infinite for every neighbourhood $V$ of $x$ in $X$.

Definition 1.1. (i) Let $\mathcal{U}=\left\{U_{n}: n \in \mathbb{N}\right\}$ be a sequence of sets. If $x_{n} \in U_{n}$ for every $n \in \mathbb{N}$, we shall call the sequence $\left\{x_{n}: n \in \mathbb{N}\right\}$ a selection for $\mathcal{U}$.

(ii) A topological space $X$ is selectively pseudocompact if every sequence $\left\{U_{n}: n \in \mathbb{N}\right\}$ of nonempty open subsets of $X$ admits a selection $\left\{x_{n}: n \in \mathbb{N}\right\}$ which has an accumulation point in $X$.

This notion was introduced by García-Ferreira and Ortiz-Castillo [9] under the name "strong pseudocompactness". The present name and an equivalent reformulation of the property given in item (ii) of Definition 1.1 is due to Dorantes-Aldama and the first author [7, Theorem 2.1 and

The first listed author was partially supported by the Grant-in-Aid for Scientific Research (C) No. 26400091 of the Japan Society for the Promotion of Science (JSPS).

This paper was written as part of the second listed author's Master's Program at the Graduate School of Science and Engineering of Ehime University. The second listed author was partially supported by the 2016-2017 fiscal year grant of the Matsuyama Saibikai. 
Definition 2.2]. One easily sees that

countably compact $\rightarrow$ selectively pseudocompact $\rightarrow$ pseudocompact.

In 2015, García-Ferreira and Tomita constructed a Boolean pseudocompact group that is not selectively pseudocompact [10, Example 2.4]. In this paper we establish a fairly general result involving the Bohr topology on all countable subgroups of a given topological group (Theorem 4.1) from which we deduce that many known examples in the literature of pseudocompact Boolean groups automatically fail to be selectively pseudocompact. In particular, each of the strongly selfdual pseudocompact groups constructed by Tkachenko [16, Theorem 3.3] in 2009 is not selectively pseudocompact either. Therefore, even strongly self-dual pseudocompact Boolean groups need not be selectively pseudocompact (Corollary 5.8). Furthermore, pseudocompact group topologies with property $\sharp$ on Boolean groups constructed by Galindo and Macario [8] in 2011 are also not selectively pseudocompact. Based on this, we show that, under the Singular Cardinal Hypothesis $\mathrm{SCH}$, every pseudocompact Boolean group admits a pseudocompact group topology which fails to be selectively pseudocompact (Corollary [5.4).

\section{Preliminaries}

Every abelian group $G$ has the maximal precompact group topology on $G$ called its Bohr topology. This topology is simply the initial topology with respect to the family of all homomorphisms from $G$ to the circle group $\mathbb{T}$. We shall use $G^{\#}$ to denote the abelian group $G$ endowed with its Bohr topology.

Definition 2.1. [15] A subgroup $H$ of a topological group $G$ is said to be $h$-embedded in $G$ if every homomorphism from $H$ to the circle group $\mathbb{T}$ is a restriction of some continuous group homomorphism from $G$ to $\mathbb{T}$.

The next fact and its corollary are part of folklore.

Fact 2.2. If $H$ is an h-embedded subgroup of an abelian topological group $G$, then the subspace topology on $H$ induced by the topology of $G$ is finer than the Bohr topology of $H$.

Proof. Since the Bohr topology of $H$ is the initial topology with respect to the family of all homomorphisms from $H$ to the circle group $\mathbb{T}$, the conclusion easily follows from Definition 2.1.

Corollary 2.3. If $G$ is an abelian topological group such that every countable subgroup of $G$ is $h$-embedded in $G$, then the subspace topology on each countable subgroup $H$ of $G$ induced by the topology of $G$ is finer than the Bohr topology of $H$.

The class of abelian topological groups $G$ such that every countable subgroup of $G$ is $h$-embedded in $G$ plays a prominent role in Pontryagin duality theory, as witnessed by Fact 5.1 below.

Remark 2.4. The authors do not know if the converse implications in Fact 2.2 and Corollary 2.3 hold.

Remark 2.5. If all countable subgroups of a topological group $G$ are closed in $G$, then the closure of every countable subset of $G$ is countable. Indeed, let $X$ be a countable subset of $G$. Then the subgroup $\langle X\rangle$ of $G$ is also countable, so it is closed in $G$ by our assumption. Since $X \subseteq\langle X\rangle$, the closure of $X$ in $G$ is contained in the countable set $\langle X\rangle$, so it is countable.

Fact 2.6. [4, §2] If $G$ is an abelian group, then every subgroup $H$ of $G$ is closed in $G^{\#}$.

Proposition 2.7. Let $G$ be a topological abelian group such that the subspace topology on each countable subgroup $H$ of $G$ induced by the topology of $G$ is finer than the Bohr topology of $H$. Then:

(i) all countable subgroups of $G$ are closed, and 
(ii) all separable pseudocompact subsets of $G$ are finite.

Proof. (i) Let $C$ be a countable subgroup of $G$. To prove that $C$ is closed in $G$, we fix an arbitrary element $g \in G \backslash C$ and find an open neighbourhood $V$ of $g$ disjoint from $C$. Let $H=\langle C \cup\{g\}\rangle$. Note that $C$ is closed in $H^{\#}$ by Fact 2.6, so we can fix an open neighbourhood $U$ of $g$ in $H^{\#}$ disjoint from $C$. Since $H$ is countable, by our assumption, the subspace topology on $H$ is finer than the Bohr topology of $H$. Therefore, we can find an open subset $V$ of $G$ such that $V \cap H=U$. Since $g \in U$ and $U \cap C=\emptyset$, it follows that $V$ is an open neighbourhood of $g$ in $G$ disjoint from $C$.

(ii) By (i), all countable subgroups of $G$ are closed in $G$, so we can apply Remark 2.5 to conclude that the closure of each countable subset of $G$ is countable. Let $X$ be in infinite separable pseudocompact subspace of $G$. By [14, Lemma 10], $X$ contains a non-trivial convergent sequence. The subgroup $K=\langle X\rangle$ of $G$ is countable. By the assumption of our proposition, the Bohr topology of $K$ is coarser than the subspace topology of $K$ inherited from $G$. Since $X$ is a convergent sequence in $K$, it must be a convergent sequence in $K^{\#}$. This contradicts the well-known fact that the Bohr topology does not have non-trivial convergent sequences [11] (see Remark 2.11 below for more general result).

Combining Corollary 2.3 with Proposition 2.7, we obtain the following

Corollary 2.8. If all countable subgroups of an abelian group $G$ are h-embedded in $G$, then all separable pseudocompact subsets of $G$ are finite.

Remark 2.9. (1) Hernández and Macario [13] say that a space $X$ is countably pseudocompact if every countable subset of $X$ is contained in a separable pseudocompact subset of $X$. A space $X$ is said to be countably pracompact if $X$ contains a dense set $Y$ such that every infinite subset of $Y$ has an accumulation point in $X$; see [2, Ch. III, Sec. 4].

(2) As was noted in the text prior to [14, Remark 2], if all separable pseudocompact subsets of a topological space $X$ are finite, then $X$ does not contain infinite subsets which are either countably pseudocompact or countably pracompact; in particular, $G$ does not contain infinite countably compact subsets.

Remark 2.10. (a) In [1, Proposition 2.1], Ardanza-Trevijano, Chasco, Domínguez and Tkachenko proved the following result. Let $G$ be a topological abelian group such that every countable subgroup of $G$ is h-embedded in $G$. Then all countable subgroups of $G$ are closed, all compact subsets of $G$ are finite, and $G$ is sequentially closed in its completion.

(b) Corollary 2.3 shows that the assumption of Proposition 2.7 is (potentially) weaker than that of the statement in item (a).

(c) It follows from Remark 2.9 (2) that item (ii) of Proposition 2.7 strengthens the second conclusion of the statement quoted in (a). In particular, Corollary 2.8 strengthens the second conclusion of the statement in item (a).

Remark 2.11. Item (ii) of Proposition 2.7 should be compared with [6. Corollary 6.4] which says that the Bohr topology of an abelian group does not have infinite pseudocompact subsets. Indeed, it is easy to see that every subgroup $H$ of the topological group $G^{\#}$ is $h$-embedded in $G^{\#}$ and inherits from $G^{\#}$ its Bohr topology. This means that every abelian group $G$ equipped with its Bohr topology satisfies the assumptions of Proposition 2.7 and its Corollary 2.8. Therefore, the non-existence of infinite separable pseudocompact subsets in item (ii) of Proposition 2.7 can be viewed as the best possible conclusion under the much weaker assumptions of Proposition 2.7 and Corollary 2.8. That the word "separable" cannot be omitted from item (ii) of Proposition 2.7 and its Corollary 2.8 will be seen from numerous examples of pseudocompact groups satisfying the assumptions of these two results exhibited in Section 5 .

A subset $X$ of an abelian group $G$ is independent if $0 \notin X$ and $\langle A\rangle \cap\langle X \backslash A\rangle=\{0\}$ for every subset $A$ of $X$. 
We shall need the following result of Hart and van Mill shown in [12, Lemma 1.4].

Fact 2.12. Every independent subset of an abelian group $G$ is closed and discrete in $G^{\#}$.

A straightforward proof of the following folklore fact is left to the reader.

Fact 2.13. A subset $X$ of a Boolean group is independent if and only if $\sum_{a \in A} a \neq 0$ for every non-empty finite subset $A$ of $X$.

\section{An AUXiliary CONSTRUCTION}

This section is inspired by the proof of [9, Lemma 2.1].

Even though all results in this section hold for arbitrary (not necessarily commutative) topological groups $G$, we shall use abelian notations denoting the group operation of $G$ by + and its identity element by 0 .

Lemma 3.1. Let $G$ be a non-discrete topological group and $V_{-1}=G$. Then there exist a sequence of points $\left\{g_{n}: n \in \mathbb{N}\right\} \subseteq G \backslash\{0\}$ and a sequence $\left\{V_{n}: n \in \mathbb{N}\right\}$ of open neighbourhoods of 0 such that the following conditions hold for every $n \in \mathbb{N}$ :

$\left(\alpha_{n}\right) 0 \notin g_{n}+V_{n}+V_{n} \subseteq V_{n-1}$,

$\left(\beta_{n}\right) V_{n} \subseteq V_{n-1}$.

Proof. By induction on $n \in \mathbb{N}$, we shall select $g_{n} \in G \backslash\{0\}$ and an open neighbourhood $V_{n}$ of 0 satisfying $\left(\alpha_{n}\right)$.

Fix $n \in \mathbb{N}$. Suppose that $g_{i} \in G_{i} \backslash\{0\}$ and an open neighbourhood $V_{i}$ of 0 satisfying $\left(\alpha_{i}\right)$ and $\left(\beta_{i}\right)$ have already been chosen for every $i=0, \ldots, n-1$. Since $V_{n-1}$ is an open neighbourhood of 0 and $G$ is non-discrete, we have $V_{n-1} \neq\{0\}$. Then $U=V_{n-1} \backslash\{0\}$ is a non-empty open subset of $G$. Thus, we can select some $g_{n} \in U$. Using the continuity of the group operation of $G$, we can find an open neighbourhood $V_{n}$ of 0 such that $g_{n}+V_{n}+V_{n} \subseteq U$ and $V_{n} \subseteq V_{n-1}$. Now $\left(\alpha_{n}\right)$ and $\left(\beta_{n}\right)$ hold.

Lemma 3.2. Under the assumptions of Lemma 3.1,

$$
\sum_{j=1}^{k}\left(g_{i_{j}}+V_{i_{j}}\right)+V_{i_{k}} \subseteq g_{i_{1}}+V_{i_{1}}+V_{i_{1}}
$$

for every strictly increasing finite sequence $i_{1}, \ldots, i_{k} \in \mathbb{N}$.

Proof. We prove this lemma by induction on the length $k$ of the sequence. Note that (11) trivially holds for sequences of length 1. Suppose that (1) has been verified for all strictly increasing sequences of length $k$, and let $i_{1}, \ldots, i_{k+1} \in \mathbb{N}$ be a strictly increasing sequence. Observe that

$$
\sum_{j=1}^{k+1}\left(g_{i_{j}}+V_{i_{j}}\right)+V_{i_{k+1}} \subseteq \sum_{j=1}^{k}\left(g_{i_{j}}+V_{i_{j}}\right)+g_{i_{k+1}}+V_{i_{k+1}}+V_{i_{k+1}} \subseteq \sum_{j=1}^{k}\left(g_{i_{j}}+V_{i_{j}}\right)+V_{i_{k+1}-1}
$$

by $\left(\alpha_{i_{k+1}}\right)$. Since $i_{k}<i_{k+1}$, from $\left(\beta_{i_{k}+1}\right),\left(\beta_{i_{k}+2}\right), \ldots,\left(\beta_{i_{k+1}-1}\right)$ we obtain

$$
V_{i_{k}} \supseteq V_{i_{k}+1} \supseteq \cdots \supseteq V_{i_{k+1}-1} .
$$

By inductive hypothesis, (11) holds. Combining (11), (2) and (3), we get

$$
\sum_{j=1}^{k+1}\left(g_{i_{j}}+V_{i_{j}}\right)+V_{i_{k+1}} \subseteq \sum_{j=1}^{k}\left(g_{i_{j}}+V_{i_{j}}\right)+V_{i_{k+1}-1} \subseteq \sum_{j=1}^{k}\left(g_{i_{j}}+V_{i_{j}}\right)+V_{i_{k}} \subseteq g_{i_{1}}+V_{i_{1}}+V_{i_{1}} .
$$

This finishes the inductive step. 
Corollary 3.3. Each non-discrete group $G$ has a sequence $\left\{U_{n}: n \in \mathbb{N}\right\}$ of non-empty open subsets of $G$ such that

$$
0 \notin \sum_{j=1}^{k} U_{i_{j}}
$$

for every strictly increasing finite sequence $i_{1}, \ldots, i_{k} \in \mathbb{N}$.

Proof. Consider the sequences $\left\{g_{n}: n \in \mathbb{N}\right\}$ and $\left\{V_{n}: n \in \mathbb{N}\right\}$ as in the conclusion of Lemma 3.1 Let $U_{n}=g_{n}+V_{n}$ for every $n \in \mathbb{N}$. Clearly, $\mathcal{U}=\left\{U_{n}: n \in \mathbb{N}\right\}$ is a sequence of non-empty open subsets of $G$.

Let $i_{1}, \ldots, i_{k} \in \mathbb{N}$ be a strictly increasing finite sequence. Then

$$
\sum_{j=1}^{k} U_{i_{j}}=\sum_{j=1}^{k}\left(g_{i_{j}}+V_{i_{j}}\right) \subseteq \sum_{j=1}^{k}\left(g_{i_{j}}+V_{i_{j}}\right)+V_{i_{k}} \subseteq g_{i_{1}}+V_{i_{1}}+V_{i_{1}}
$$

by $0 \in V_{i_{k}}$ and Lemma 3.2. Since $0 \notin g_{i_{1}}+V_{i_{1}}+V_{i_{1}}$ by $\left(\alpha_{i_{1}}\right)$, this implies (4).

\section{MAin RESUlt}

We are now ready to state and prove our main result.

Theorem 4.1. Let $G$ be an infinite Boolean topological group such that the subspace topology on every countable subgroup $H$ of $G$ is finer than the Bohr topology of $H$. Then $G$ is not selectively pseudocompact.

Proof. Suppose that $G$ is discrete. Since $G$ is infinite, it is not pseudocompact, and thus $G$ is not selectively pseudocompact either.

From now on we shall suppose that $G$ is non-discrete. Let $\mathcal{U}=\left\{U_{n}: n \in \mathbb{N}\right\}$ be the sequence of non-empty open subsets of $G$ as in Corollary 3.3, and let $\left\{x_{n}: n \in \mathbb{N}\right\}$ be an arbitrary selection for $\mathcal{U}$. According to item (ii) of Definition 1.1, in order to prove that $G$ is not selectively pseudocompact, it suffices to show that the sequence $\left\{x_{n}: n \in \mathbb{N}\right\}$ does not have an accumulation point in $G$. This will be established in Claim 2 below.

Claim 1. $X=\left\{x_{n}: n \in \mathbb{N}\right\}$ is a faithfully indexed independent subset of $G$.

Proof. First, we check that $X$ is faithfully indexed. Let $m, n \in \mathbb{N}$ and $m<n$. Then $0 \notin U_{m}+U_{n}$ by Corollary [3.3. On the other hand, $x_{m}+x_{n} \in U_{m}+U_{n}$, so $x_{m}+x_{n} \neq 0$. Since $G$ is a Boolean group, this implies $x_{m} \neq x_{n}$.

Next, we check that $X$ is independent. By Fact 2.13, it suffices to show that $\sum_{j=1}^{k} x_{i_{j}} \neq 0$ for every faithfully indexed finite subset $\left\{i_{j}: j=1, \ldots, k\right\}$ of $\mathbb{N}$. Without loss of generality, we may assume that the sequence $i_{1}, \ldots, i_{k}$ is strictly increasing. Since $\sum_{j=1}^{k} x_{i_{j}} \in \sum_{j=1}^{k} U_{i_{j}}$, from Corollary 3.3 we conclude that $\sum_{j=1}^{k} x_{i_{j}} \neq 0$.

Claim 2. The sequence $\left\{x_{n}: n \in \mathbb{N}\right\}$ does not have an accumulation point in $G$.

Proof. Let $g \in G$ be arbitrary, and let $X$ be the set as in Claim 1, The subgroup $H=\langle X \cup\{g\}\rangle$ of $G$ is countable. Since $X$ is an independent subset of $G$ by Claim 1, it is also an independent subset of $H$, as $H$ is a subgroup of $G$ containing $X$. By Fact 2.12, $X$ is closed and discrete in $H^{\#}$. Since $g \in H$, we can find an open neighbourhood $U$ of $g$ in $H^{\#}$ such that $U \cap X \subseteq\{g\}$. Since the subspace topology on $H$ is finer than its Bohr topology by our assumption on $G$, the set $U$ is open in $H$. Thus, there exists an open subset $V$ of $G$ such that $V \cap H=U$. Since $X \subseteq H$, we get $V \cap X=V \cap H \cap X=U \cap X \subseteq\{g\}$. Since the set $X$ is faithfully indexed by Claim 1, this means that $g$ cannot be an accumulation point of the sequence $\left\{x_{n}: n \in \mathbb{N}\right\}$. Since $g \in G$ was taken arbitrarily, this means that the sequence $\left\{x_{n}: n \in \mathbb{N}\right\}$ does not have an accumulation point in $G$. 
From Corollary 2.3 and Theorem 4.1, one obtains the following

Corollary 4.2. Let $G$ be an infinite Boolean group all countable subgroups of which are h-embedded in $G$. Then $G$ is not selectively pseudocompact.

The rest of the paper is devoted to applications of Corollary 4.2 aimed at establishing the abundance of pseudocompact abelian groups which are not selectively pseudocompact.

\section{Applichtions of the main Result}

For an abelian topological group $G$, we denote by $G^{\wedge}$ the group of all continuous group homomorphisms from $G$ to $\mathbb{T}$ endowed with the compact-open topology. Recall that $G^{\wedge}$ is called the Pontryagin dual of $G$. For each $g \in G$, the map $\psi_{g}: G^{\wedge} \rightarrow \mathbb{T}$ defined by $\psi_{g}(h)=h(g)$ for every $h \in G^{\wedge}$, is a continuous homomorphism from $G^{\wedge}$ to $\mathbb{T}$, so $\psi_{g} \in G^{\wedge \wedge}$; that is, $\psi_{g}$ is an element of the second dual $G^{\wedge \wedge}$ of $G$. Consider the map $\alpha_{G}: G \rightarrow G^{\wedge \wedge}$ defined by $\alpha_{G}(g)=\psi_{g}$ for $g \in G$. The group $G$ is called (Pontryagin) reflexive when $\alpha_{G}$ is a topological isomorphism between $G$ and $G^{\wedge \wedge}$.

The following result is very useful for constructing examples of reflexive groups. It follows from [8, Lemma 2.3 and Theorem 6.1] and can be found explicitly in [1, Theorem 2.8].

Fact 5.1. If all countable subgroups of a pseudocompact abelian group $G$ are h-embedded in $G$, then $G$ is reflexive.

The following result of Galindo and Macario attests to an abundance of pseudocompact reflexive abelian groups $G$ having the property that all countable subgroups of $G$ are $h$-embedded in $G$.

Fact 5.2. Let $G$ be an infinite pseudocompact abelian group. Assume also that one of the following two conditions is satisfied:

(i) $|G| \leq 2^{2^{\mathrm{c}}}$;

(ii) the Singular Cardinal Hypothesis SCH holds.

Then $G$ admits a pseudocompact group topology $\tau$ such that every countable subgroup of $(G, \tau)$ is $h$-embedded in it. In particular, $(G, \tau)$ is a reflexive group and all compact subsets of $(G, \tau)$ are finite.

Proof. By [8, Corollary 5.6 and Theorem 5.8], $G$ admits a pseudocompact group topology $\tau$ such that every countable subgroup of $(G, \tau)$ is $h$-embedded in it (topological groups with this property are said to have property $\sharp$ in $[8$, Definition 2.2$])$. The reflexivity of $(G, \tau)$ follows from Fact 5.1 , By Remark 2.10 (a), all compact subsets of $(G, \tau)$ are finite.

Remark 5.3. It follows from Corollary 2.8 and Remark 2.9 (2) that the topology $\tau$ in Fact 5.2 has the stronger property that all separable pseudocompact subsets of $(G, \tau)$ are finite.

Corollary 5.4. An infinite pseudocompact Boolean group $G$ admits a pseudocompact reflexive group topology which is not selectively pseudocompact in each of the following cases:

(i) $|G| \leq 2^{2^{\mathfrak{c}}}$;

(ii) the Singular Cardinal Hypothesis SCH holds.

Proof. Let $G$ be an infinite pseudocompact Boolean group. Apply Fact 5.2 to find a pseudocompact reflexive group topology $\tau$ on $G$ such that every countable subgroup of $(G, \tau)$ is $h$-embedded in it. Finally, $(G, \tau)$ is not selectively pseudocompact by Corollary 4.2 .

It follows from Corollary 5.4 that, under the assumption of $\mathrm{SCH}$, every pseudocompact Boolean group $G$ can be equipped with a (reflexive) pseudocompact group topology which fails to be selectively pseudocompact. Moreover, for "small" groups $G$ the assumption of SCH is superfluous. 
Note that SCH is a "rather mild" additional set-theoretic assumption beyond ZFC, the ZermeloFraenkel axioms of set theory augmented by the Axiom of Choice. Indeed, the failure of SCH implies the existence of a large cardinal [5]. Nevertheless, we do not know if one can omit the Singular Cardinal Hypothesis SCH completely; see Question 6.2.

An abelian topological group $G$ is called self-dual if $G$ is topologically isomorphic to its Pontryagin dual $G^{\wedge}$.

Corollary 5.5. An infinite self-dual pseudocompact Boolean group cannot be selectively pseudocompact.

Proof. Let $G$ be a self-dual pseudocompact Boolean group. By [16, Theorem 2.3], all countable subgroups of $G$ are $h$-embedded in $G$, so $G$ is not selectively pseudocompact by Corollary 4.2.

We refer the reader to [16] for the definition of strong self-duality. As can be guessed from the terminology, strongly self-dual abelian topological groups are self-dual. By [16, Proposition 2.2], strongly self-dual abelian topological groups are reflexive.

Finally, we recall the result of Tkachenko shown in [16, Theorem 3.3].

Fact 5.6. Let $\kappa$ be an infinite cardinal with $\kappa^{\omega}=\kappa$. Then there exists a pseudocompact strongly self-dual Boolean group $G$ satisfying $|G|=w(G)=\kappa$.

Corollary 5.7. For every infinite cardinal $\kappa$ satisfying $\kappa^{\omega}=\kappa$, there exists a pseudocompact non-selectively pseudocompact strongly self-dual Boolean group $G$ such that $|G|=w(G)=\kappa$.

Proof. Let $\kappa$ be an infinite cardinal such that $\kappa^{\omega}=\kappa$. Apply Fact 5.6 to find a pseudocompact strongly self-dual Boolean group $G$ satisfying $|G|=w(G)=\kappa$. Then $G$ is self-dual, so $G$ cannot be selectively pseudocompact by Corollary 5.5 .

Corollary 5.8. A strongly self-dual pseudocompact Boolean group need not be selectively pseudocompact.

\section{Open questions}

It follows from Fact 5.2 (ii) and Remark 5.3 that, under the assumption of SCH, every infinite pseudocompact abelian group $G$ admits a pseudocompact group topology $\tau$ such that all separable pseudocompact subsets of $(G, \tau)$ are finite. It is not clear whether this result can be proved without any additional set-theoretic assumptions beyond ZFC.

Question 6.1. Is it true in ZFC that every infinite pseudocompact abelian group $G$ admits a pseudocompact group topology $\tau$ such that all separable pseudocompact subsets of $(G, \tau)$ are finite?

It follows from Fact 5.2 (i) that the answer to this question is positive when $|G| \leq 2^{2^{\mathrm{c}}}$.

Question 6.2. Is it true in ZFC that every pseudocompact Boolean group admits a pseudocompact non-selectively pseudocompact group topology? Can this topology be made also reflexive?

As was noted after Corollary 5.4, the answer to this question is positive under SCH.

It is unclear whether Corollary 5.4 holds beyond the class of Boolean groups.

Question 6.3. Does every pseudocompact abelian group admit a pseudocompact group topology which is not selectively pseudocompact? Can this topology be made also reflexive?

\section{REFERENCES}

[1] S. Ardanza-Trevijano, M. J. Chasco, X. Domínguez, M. Tkachenko, Precompact noncompact reflexive Abelian groups, Forum Math. 24 (2012) 289-302.

[2] A.V. Arhangelskii, Topological Function Spaces, Math. Appl. (Soviet Ser.), vol. 78, Kluwer, Dordrecht, 1992. 
[3] W.W. Comfort, K.A. Ross, Pseudocompactness and uniform continuity in topological groups, Pacific J. Math. 16 (3) (1966) 483-496.

[4] W. W. Comfort, V. Saks, Countably compact groups and finest totally bounded topologies, Pacific J. Math. 49 (1973) 33-44.

[5] K. I. Devlin, R. B. Jensen, Marginalia to a theorem of Silver, ISILC Logic Conference, Springer Lectures Notes 499 (1975) 115-142.

[6] D. Dikranjan, D. Shakhmatov, A Kronecker-Weyl theorem for subsets of abelian groups, Adv. Math. 226 (2011) 4776-4795.

[7] A. Dorantes-Aldama, D. Shakhmatov, Selective sequential pseudocompactness, Topology Appl. 222 (2017) $53-69$.

[8] J. Galindo and S. Macario, Pseudocompact group topologies with no infinite compact subsets, J. Pure Appl. Algebra 215 (2011) 655-663.

[9] S. García-Ferreira, Y. F. Ortiz-Castillo, Strong pseudocompact properties, Comment. Math. Univ. Carol. 55 (2014) 101-109

[10] S. García-Ferreira, A. H. Tomita, A pseudocompact group which is not strongly pseudocompact, Topology Appl. 192 (2015) 138-144.

[11] I. Glicksberg, Uniform boundedness for groups, Canad. J. Math. 14 (1962) 269-276.

[12] K. P. Hart, J. van Mill, Discrete sets and the maximal totally bounded group topology, Journal of Pure and Applied Algebra 70 (1991) 73-80.

[13] S. Hernández, S. Macario, Dual properties in totally bounded Abelian groups, Arch. Math. 80 (2003) $271-283$.

[14] D. Shakhmatov, V. H. Yañez, Selectively pseudocompact groups without infinite separable pseudocompact subsets, Axioms 7(4), 86 (2018) 23 pp., doi:10.3390/axioms7040086.

[15] M. G. Tkačenko, Compactness type properties in topological groups, Czechoslovak Math. J. 38 (113(2)) (1988) 324-341.

[16] M. Tkachenko, Self-duality in the class of precompact groups, Topology Appl. 156 (2009) 2158-2165.

Division of Mathematics, Physics and Earth Sciences, Graduate School of Science and Engineering, Ehime University, MATSUyAma 790-8577, JAPAN

E-mail address: dmitri.shakhmatov@ehime-u.ac.jp

Master's Course, Graduate School of Science and Engineering, Ehime University, Matsuyama 7908577, JAPAN

E-mail address: victor_yanez@comunidad.unam.mx 